\title{
Factores asociados a la actitud del profesional de enfermería ante la muerte del paciente terminal
}

\author{
Mirabel García Rojas, ${ }^{1, a, b}$
}

García RM. Factores asociados a la actitud del profesional de enfermería ante la muerte del paciente terminal. Cuid salud, ene-jun 2017; 3(1):277-287.

\section{RESUMEN}

Objetivo: determinar los factores asociados a la actitud del profesional de Enfermería, ante la muerte del paciente terminal. Metodología: estudio cuantitativo, diseño descriptivo correlacional; realizado en servicios de cuidados intensivos (UCIs) de un hospital nacional de categoría III-1. Todas las enfermeras formaron parte del estudio $(\mathrm{N}=65)$, quienes respondieron dos formularios mediante encuesta: 1) medición de factores y 2) medición de la actitud a través del CAM-1 adaptado. La recolección de datos fue realizada en octubre-2015; analizándose los datos mediante la estadística descriptiva y la prueba de Chi cuadrado para correlacionar las variables. Resultados: el 55\% de enfermeras tenía edad entre 31- 40 años, predominando el sexo femenino, con formación en cuidados intensivos; la mayoría tenía contrato laboral y recientemente había sido incorporada al servicio; la relación enfermera-paciente fue de 1-2; destacando que más del 50\% trabajaba en otras instituciones. Acerca de la actitud, predominó la indiferencia en las enfermeras, existiendo relación entre actitud indiferente ante la muerte con el tipo de UCI y trabajar en otra institución. Conclusión: algunos factores laborales se relacionan con la actitud indiferente del profesional de enfermería ante la muerte del paciente terminal; situación que debe ser abordado por las instituciones para ofrecer una asistencia adecuada.

Palabras clave: atención al paciente, actitud frente la muerte, espiritualidad, humanización de la atención, enfermería (Fuente: DeCS BIREME).
García RM. Factors associated with nursing professional's attitude at the death of terminally ill patients. Cuid salud, ene-jun 2017; 3(1):277-287.

\begin{abstract}
Objective: To determine the factors associated with the attitude of the nursing professional, in view of the death of the terminally ill patient. Methodology: Quantitative study, descriptive correlational design; performed in intensive care services (ICUs) of a category III-1 national hospital. All nurses were part of the study $(\mathrm{N}=$ 65), who answered two forms through a survey: 1) factor measurement and 2) attitude measurement through the adapted CAM-1. Data collection was done in October-2015; analyzing the data using descriptive statistics and Chi square test to correlate the variables. Results: $55 \%$ of nurses were aged between 31-40 years, predominantly female, with intensive care training; most had a work contract and had recently been incorporated into the service; the nurse-patient ratio was 1-2; noting that more than $50 \%$ worked in other institutions. About the attitude, the indifference in the nurses predominated, existing relationship between indifferent attitude towards death with the type of ICU and working in another institution. Conclusion: Some labor factors are related to the indifferent attitude of the nursing professional to the death of the terminal patient; a situation that must be addressed by the institutions to provide adequate assistance.
\end{abstract}

Keywords: Patient care, attitude to death, spirituality, humanization of assistance, nursing (Source: MeSH NLM).

\footnotetext{
1 Congregación Religiosa San Vicente de Paul

a Licenciada Enfermera.

b Egresada de la Escuela de Enfermería Padre Luis Tezza afiliada a la Universidad Ricardo Palma, Lima-Perú.
} 


\section{INTRODUCCIÓN}

La muerte es un fenómeno natural inevitable que afectará a todos los miembros de la especie, más pronto o más tarde, incluido a los médicos y enfermeras. ${ }^{1}$ Sin embargo, en las sociedades industrializadas cada vez es más difícil el convivir o aceptar la mera idea de la muerte según Sontag, citado por Mazza et al., ${ }^{2}$ arraigado al avance de la ciencia y tecnología que ha conllevado al personal de salud a luchar contra la muerte y el dolor.

Mismo así, el manejo de la muerte es muy complejo dentro de las instituciones de salud, principalmente en aquellos servicios de atención a personas con enfermedades crónico-degenerativas y donde la muerte del paciente forma parte de lo cotidiano, haciendo necesario valorar la muerte no únicamente como un proceso biológico, sino también como proceso psicológico, cultural y económico. ${ }^{3}$ Pues, tanto la idea como la consciencia de muerte son características exclusivamente humanas que provocan emociones intensas y una gama de actitudes y creencias que se expresan mediante distintas reacciones como desesperación, miedo y ansiedad. ${ }^{4}$

Actualmente, muchas personas mueren al final de un penoso período de enfermedad crónica, luego del deterioro funcional progresivo de su organismo, en cuya situación el tratamiento curativo es sustituido por los cuidados paliativos, que tienen entre sus objetivos prepararse para la muerte del paciente, tanto al enfermo mismo como a sus familiares, ${ }^{5}$ además de prevenir y aliviar el sufrimiento mediante la detección temprana del dolor u otros problemas de orden físico, psicosocial o espiritual. ${ }^{6}$

Según Sepulveda, ${ }^{7}$ alrededor de 35 millones de pacientes en el mundo requieren de cuidados paliativos, identificándose en esta condición a personas con enfermedades crónicas potencialmente mortales como el cáncer, VIH, insuficiencia cardiaca congestiva, enfermedades cerebrovasculares, enfermedades neurodegenerativas, entre otras.

Kübler-Ross, citado por Oviedo et al., ${ }^{8}$ describe que el paciente y su familia atraviesan por cinco fases desde el momento en que reconocen que la muerte es inminente, iniciando con la negación, ira, negociación, depresión y aceptación. Fases en que requieren de mucho apoyo presencial y moral para ajustarse y aceptar el desprendimiento de la vida, la separación y la muerte en sí misma. Confrontación que resulta dolorosa y difícil para todos, incluso para el profesional de salud, quien debe mostrar una actitud serena y equilibrada para aliviar sus sentimientos de tensión y cubrir al mismo tiempo las necesidades del paciente con enfermedad terminal. $^{9}$

El profesional de enfermería tiene dentro de sus funciones, la de ayudar a los seres humanos a enfrentar este paso de transición de la vida a la muerte, tanto al moribundo como a los seres queridos que lo rodean, esperándose de ella una actitud cálida, favorable y de apoyo con el necesitado. ${ }^{2}$ Actitud que dependerá mucho de su preparación, de su experiencia y de sus creencias religiosas o agnósticas, así como de su capacidad para enfrentarse con la realidad de la vida o de la muerte; lo que indica directamente en cómo se plantea el acompañamiento de los otros seres humanos que mueren. $^{3}$

Maza et al. $^{2}$ refieren que Enfermería se enfrenta cotidianamente con la muerte y tal vez sin recibir apoyo institucional para saber lidiar con sus sentimientos de miedo y angustia, evitando siempre el tema de muerte; lo que puede conllevar a una pobre atención del paciente terminal. Al respecto, Sánchez ${ }^{10}$ acota que los cuidados enfermeros no siempre reflejan su naturaleza global y holístico, dado que las enfermeras (os) se muestran distantes del paciente y su familia en el trato, con respuestas inadecuadas, gestos de impaciencia, poca tolerancia y muchas veces con alto grado de deshumanización.

Diversos estudios sobre las actitudes del profesional de enfermería frente al cuidado del paciente terminal revelan indiferencia y rechazo $\mathrm{o}$ negatividad, ${ }^{11,12}$ desde una perspectiva fisiológico, espiritual y psicoemocional. $^{12}$ Entre los factores relacionados con esta actitud resaltan a la edad, haber recibido formación sobre la muerte en pregrado, edad del primer contacto con el deceso y años de experiencia profesional. $^{2}$

En el Perú, los cuidados paliativos datan de 1989, con la primera unidad de dolor y cuidados paliativos al paciente con cáncer en el Hospital de Policía. ${ }^{13}$ Hecho que ha evolucionado desde entonces, sobre todo en la preparación del personal que labora en esta área. Entre tanto, el poder de la técnica y la tecnología han creado nuevas exigencias administrativas en el cuidado, suplantándolo con el seguimiento y control de procesos estandarizados y 
sistematizados; tornando el cuidado burocrático en deterioro de la relación comprensiva y afectuosa entre paciente-enfermera. ${ }^{14}$ Rivera ${ }^{15}$ señala que los pacientes también desean ser auxiliados espiritualmente para encontrarse con el ser sobrenatural, con los demás y consigo mismo. Situación que se torna crítica en el paciente terminal, cuyo acompañamiento espiritual es elemento fundamental del cuidado enfermero.

El cuidado debe ser preferentemente humano, reconociendo la unicidad de la persona y el cuidado como ciencia y arte, con actitudes humanas y espirituales, rasgo que evidencia el rol invalorable de la enfermera. Quintero ${ }^{16}$ refiere que el cuidado involucra el componente afectiva y expresa una forma de amar, posibilitando la expresión de sentimientos y emociones traducidos en el hacer y en las actitudes.

Por lo tanto, reconociendo la importancia de las competencias emocionales que requiere la enfermera para brindar apoyo al paciente terminal y el haberlos acompañado en la práctica hospitalaria identificando que muchos buscan consuelo y esperanza en un ser supremo, el objetivo del estudio fue determinar los factores asociados a la actitud del profesional de enfermería ante la muerte del paciente terminal en un hospital nacional de Lima-Perú.

\section{METODOLOGÍA}

Este estudio fue de enfoque cuantitativo, tipo aplicada, método no experimental y diseño descriptivo correlacional; realizado en los servicios de cuidados intensivos del Hospital Nacional Dos de Mayo, ubicado en el centro de Lima-Perú, perteneciente a la DISA V, con categoría III-1; constituyendo un hospital de referencia nacional. ${ }^{17}$

La población universo fue constituido por 65 profesionales de enfermería que laboraban en diversas Unidades de Cuidados Intensivos (UCI) Adultos, Cardiovascular y la Unidad de Tratamiento Neurológico Intensivo (UTNI). Dado el número pequeño de la población se trabajó con 60 enfermeras (población de tipo censal) considerando como criterios de inclusión; profesionales de ambos sexos, que trabajaban en la unidad durante los últimos 12 meses o más.

La técnica utilizada para recolectar los datos fue la encuesta y el instrumento dos cuestionarios, los cuales se describen a seguir:
Instrumento 1. Cuestionario de Factores Condicionantes. Datos generales, Instrucciones, Factores personales (seis preguntas) y Factores laborales (siete preguntas).

Instrumento 2. Cuestionario de Actitudes ante la Muerte (CAM-1) adaptado por Hernández ${ }^{18,5}$ del original denominado Death attitudes and self-reported health relevant behaviors de Chloé y Salovey. ${ }^{19} \mathrm{El}$ CAM-1 fue validado en Cuba $(\alpha=0,71)^{3}$ y México $(\alpha=0,73) ;{ }^{20}$ en el Perú se validó en Tacna $(\alpha=0,803){ }^{21}$ La versión se compone de 33 reactivos (ítem), distribuidas en seis dimensiones: 1) de evitación, 2) de aceptación, 3) de temor, 4) basadas en las creencias de que la muerte es un pasaje o tránsito, 5) fundamentadas en la concepción de la muerte como una salida o solución y sobre, 6) la perspectiva del profesional. Cada respuesta tiene tres opciones: $\mathrm{Ni}$ en desacuerdo, ni en acuerdo y, En desacuerdo. Destacando que desde sus inicios el instrumento fue aplicado en profesionales de la salud.

Para el estudio, previamente el instrumento fue revisado por un grupo de expertos que sugirieron adaptar algunas palabras para mayor comprensión (ítems 2, 3, 4, 5, 6, 7, 11, 15, 25, 26, 28 y 30). Se realizó la prueba piloto para comprobar su confiabilidad mediante el Alpha de Cronbach $(\alpha=0,875)$.

Para recolectar los datos se cumplieron con los procesos administrativos institucionales; coordinando con cada jefa de los servicios la aplicación de los cuestionarios; actividad desarrollada diariamente y de forma individual en los horarios de refrigerio en un tiempo aproximado de 15-20 minutos, previa firma del consentimiento informado y luego de haber dado respuesta a preguntas por parte de los profesionales. Todo el proceso de recolección de datos fue realizado por la investigadora durante tres semanas en octubre 2015. Al recibir de vuelta los cuestionarios era verificado si fueron llenados totalmente, reduciendo así su posible eliminación por falta de respuestas.

Los datos se digitaron en Programa Excel 2013, luego trasladados al paquete estadístico SPSS versión 21.0 para su análisis respectivo mediante la estadística descriptiva (frecuencias y porcentajes). Para la comprobación de la hipótesis se aplicó la estadística inferencial utilizando la prueba de Chi cuadrado considerando un nivel de significancia de $\alpha=0,05$. 
El proyecto fue evaluado por el comité de ética del área de estudio, siendo aprobado mediante Carta $\mathrm{N}^{\circ}$ 029-2015-CEIB-AI-OACDI-HNDM, cumpliendo con la normativa nacional para estudios observacionales y que responden a los principios éticos internacionales. Durante la recolección de datos se respetaron los principios éticos de: Autonomía, beneficencia, no maleficencia y justicia; expresando que no habría ningún beneficio económico y que los datos serán manejados de manera confidencial.

\section{RESULTADOS}

De las 65 enfermeras se excluyeron cinco por tratarse de profesionales que estaban reemplazando al personal efectivo, identificándose que el 55\% de ellas tenía edades entre 31-40 años, con predominio del sexo femenino, solteras y sin hijos; destacando que casi la mitad tenía segunda especialidad y profesaban la religión católica (tabla 1).

Tabla 1: Distribución de factores personales en enfermeras de UCI. Hospital Nacional Dos de Mayo. Octubre 2015.

\begin{tabular}{|c|c|c|c|}
\hline Factores & Categorías & $\mathrm{n}$ & $\%$ \\
\hline \multirow[t]{4}{*}{ Edad (años) } & $25-30$ & 11 & 18 \\
\hline & $31-40$ & 33 & 55 \\
\hline & $41-50$ & 13 & 22 \\
\hline & $51-60$ & 3 & 5 \\
\hline \multirow[t]{2}{*}{ Sexo } & Femenino & 42 & 70 \\
\hline & Masculino & 18 & 30 \\
\hline \multirow[t]{3}{*}{ Formación postgrado } & Una especialidad & 29 & 48 \\
\hline & Dos especialidades & 28 & 47 \\
\hline & Maestría & 3 & 5 \\
\hline \multirow[t]{4}{*}{ Religión } & Católica & 52 & 87 \\
\hline & Cristiana & 4 & 7 \\
\hline & Evangélica & 1 & 2 \\
\hline & Otras & 3 & 4 \\
\hline \multirow[t]{4}{*}{ Estado civil } & Soltera (o) & 30 & 50 \\
\hline & Casada (o) & 24 & 40 \\
\hline & Conviviente & 5 & 8 \\
\hline & Divorciada (o) & 1 & 2 \\
\hline \multirow[t]{4}{*}{ Número de hijos } & Ninguno & 28 & 47 \\
\hline & Uno & 12 & 20 \\
\hline & Dos & 18 & 30 \\
\hline & Tres & 2 & 3 \\
\hline
\end{tabular}

Tabla 2: Distribución de factores laborales en enfermeras de UCI. Hospital Nacional Dos de Mayo. Octubre 2015.

\begin{tabular}{|c|c|c|c|}
\hline Factores laborales & Categoría & $\mathbf{n}$ & $\%$ \\
\hline \multirow[t]{2}{*}{ Condición Laboral } & Nombrada & 25 & 42 \\
\hline & Contratada & 35 & 58 \\
\hline \multirow[t]{4}{*}{ Servicio que labora } & UCIG & 17 & 28 \\
\hline & UCIN & 7 & 12 \\
\hline & UTNI & 16 & 27 \\
\hline & UCI Cardiovascular & 20 & 33 \\
\hline \multirow[t]{4}{*}{ Tiempo en la Institución } & 1 a 5 años & 32 & 53 \\
\hline & 6 a 10 años & 16 & 27 \\
\hline & 11 a 15 años & 4 & 7 \\
\hline & 16 años a más & 8 & 13 \\
\hline \multirow[t]{4}{*}{ Tiempo en el servicio } & 1 a 5 años & 40 & 67 \\
\hline & 6 a 10 años & 12 & 20 \\
\hline & 11 a 15 años & 2 & 3 \\
\hline & 16 años a más & 6 & 10 \\
\hline \multirow{4}{*}{$\begin{array}{l}\text { Número de pacientes } \\
\text { asignados }\end{array}$} & 2 pacientes & 26 & 43 \\
\hline & 3 pacientes & 22 & 37 \\
\hline & 4 pacientes & 3 & 5 \\
\hline & 5 pacientes & 9 & 15 \\
\hline \multirow[t]{2}{*}{ Turnos de Trabajo } & Fijo & 8 & 13 \\
\hline & Rotativo & 52 & 87 \\
\hline \multirow{2}{*}{$\begin{array}{l}\text { Trabaja en otra } \\
\text { Institución }\end{array}$} & $\mathrm{Si}$ & 33 & 55 \\
\hline & No & 27 & 45 \\
\hline \multirow[t]{3}{*}{ En qué lugar } & Ninguno & 27 & 45 \\
\hline & Clínicas & 29 & 48 \\
\hline & Docencia & 4 & 7 \\
\hline
\end{tabular}

En la tabla 2, se identifica que la mayoría de enfermeras estaba en condición de contratada (58\%) y $53 \%$ se había incorporado a la institución en los últimos cinco años, con mayor número de profesionales en UCI cardiovascular (33\%), teniendo en general dos pacientes a cargo durante el turno (43\%); con tipo de trabajo rotativo preferentemente $(87 \%)$. Asimismo, más de la mitad de las enfermeras trabajaba en otras instituciones (55\%), principalmente en el ámbito privado como las clínicas.

Gráfico 1: Clasificación de la actitud ante la muerte en enfermeras de UCI. Hospital Nacional Dos de Mayo. Octubre 2015.




Gráfico 2: Distribución de la actitud ante la muerte por dimensiones en enfermeras de UCI. Hospital Nacional Dos de Mayo. Octubre 2015.



En el gráfico 1 se observa predominio de la actitud indiferente hacia la muerte en las enfermeras del estudio y un importante $20 \%$ muestra actitud de rechazo. En el siguiente gráfico por dimensiones (gráfico 2), también sobresale la actitud indiferente, aunque el $32 \%$ acepta la muerte como un hecho real y el $26 \%$ lo rechaza (dimensión salida).

Tabla 3: Relación entre factores personales y actitud ante la muerte en enfermeros de UCI. Hospital Nacional Dos de Mayo. Octubre 2015.

\begin{tabular}{|c|c|c|c|c|c|c|c|c|}
\hline \multirow{3}{*}{ FACTORES PERSONALES } & \multicolumn{6}{|c|}{ Actitud ante la muerte } & \multirow{3}{*}{$\mathbf{X}^{2}$} & \multirow{3}{*}{$p$} \\
\hline & \multicolumn{2}{|c|}{ Rechazo } & \multicolumn{2}{|c|}{ Indiferencia } & \multicolumn{2}{|c|}{ Aceptación } & & \\
\hline & $\mathbf{N}$ & $\%$ & $\mathbf{N}$ & $\%$ & $\mathbf{N}$ & $\%$ & & \\
\hline \multicolumn{9}{|l|}{ Edad (años) } \\
\hline $25-30$ & 1 & 2 & 6 & 10 & 4 & 7 & \multirow{4}{*}{2,717} & \multirow{4}{*}{0,843} \\
\hline $31-40$ & 7 & 11 & 20 & 33 & 6 & 10 & & \\
\hline $41-50$ & 3 & 5 & 7 & 11 & 3 & 5 & & \\
\hline $51-60$ & 1 & 2 & 1 & 2 & 1 & 2 & & \\
\hline \multicolumn{9}{|l|}{ Sexo } \\
\hline Masculino & 5 & 8 & 9 & 15 & 4 & 7 & \multirow{2}{*}{0,993} & \multirow{2}{*}{0,1344} \\
\hline Femenino & 7 & 11 & 25 & 42 & 10 & 17 & & \\
\hline \multicolumn{9}{|l|}{ Estudios } \\
\hline Una Especialidad & 7 & 11 & 16 & 27 & 6 & 10 & \multirow{3}{*}{3,838} & \multirow{3}{*}{0,428} \\
\hline Dos Especialidades & 5 & 8 & 17 & 29 & 6 & 10 & & \\
\hline Maestría & - & - & 1 & 2 & 2 & 3 & & \\
\hline \multicolumn{9}{|l|}{ Religión } \\
\hline Católica & 12 & 21 & 30 & 50 & 10 & 17 & \multirow{4}{*}{8,261} & \multirow{4}{*}{0,220} \\
\hline Cristiana & - & - & 3 & 5 & 1 & 2 & & \\
\hline Evangélica & - & - & - & - & 1 & 2 & & \\
\hline Otras & - & - & 1 & 2 & 2 & 3 & & \\
\hline \multicolumn{9}{|l|}{ Estado Civil } \\
\hline Soltera & 7 & 11 & 16 & 27 & 7 & 11 & \multirow{4}{*}{3,147} & \multirow{4}{*}{0,790} \\
\hline Casada & 4 & 7 & 13 & 22 & 7 & 11 & & \\
\hline Conviviente & 1 & 2 & 4 & 7 & - & - & & \\
\hline Divorciada & - & - & 1 & 2 & - & - & & \\
\hline \multicolumn{9}{|l|}{ Número de hijos } \\
\hline Ninguno & 6 & 10 & 15 & 26 & 7 & 11 & \multirow{4}{*}{2,8907} & \multirow{4}{*}{0,0805} \\
\hline 1 hijo & 2 & 3 & 7 & 11 & 3 & 5 & & \\
\hline 2 hijos & 4 & 7 & 10 & 17 & 4 & 7 & & \\
\hline 3 hijos & - & - & 2 & 3 & - & - & & \\
\hline
\end{tabular}


Tabla 4: Relación entre factores laborales y actitud ante la muerte en enfermeras de UCI. Hospital Nacional Dos de Mayo. Octubre 2015.

\begin{tabular}{|c|c|c|c|c|c|c|c|c|}
\hline \multirow{3}{*}{ FACTORES LABORALES } & \multicolumn{8}{|c|}{ Actitud ante la muerte } \\
\hline & \multicolumn{2}{|c|}{ Rechazo } & \multicolumn{2}{|c|}{ Indiferencia } & \multicolumn{2}{|c|}{ Aceptación } & \multirow[t]{2}{*}{$\mathbf{X}^{2}$} & \multirow[t]{2}{*}{$p$} \\
\hline & $\mathbf{N}$ & $\%$ & $\mathbf{N}$ & $\%$ & $\mathbf{N}$ & $\%$ & & \\
\hline \multicolumn{9}{|l|}{ Condición Laboral } \\
\hline Nombrada & 9 & 15 & 13 & 22 & 3 & 5 & \multirow{2}{*}{8,010} & \multirow{2}{*}{0,0182} \\
\hline Contratada & 3 & 5 & 21 & 35 & 11 & 18 & & \\
\hline \multicolumn{9}{|l|}{ Servicio que labora } \\
\hline UCIG & 3 & 5 & 11 & 18 & 3 & 5 & \multirow{4}{*}{8,224} & \multirow{4}{*}{0,412} \\
\hline UCIN & 2 & 3 & 3 & 5 & 2 & 3 & & \\
\hline UTNI & 5 & 8 & 9 & 15 & 2 & 3 & & \\
\hline UCI Cardiovascular & 2 & 4 & 11 & 18 & 7 & 12 & & \\
\hline \multicolumn{9}{|c|}{ Años que labora en la Institución } \\
\hline 1 a 5 años & 6 & 10 & 19 & 32 & 7 & 12 & \multirow{4}{*}{3,726} & \multirow{4}{*}{0,714} \\
\hline 6 a 10 años & 3 & 5 & 7 & 12 & 6 & 10 & & \\
\hline 11 a 15 años & 1 & 2 & 3 & 5 & 0 & 0 & & \\
\hline 16 años a más & 2 & 3 & 5 & 8 & 1 & 2 & & \\
\hline \multicolumn{9}{|l|}{ Tiempo en el Servicio } \\
\hline 1 a 5 años & 9 & 15 & 23 & 38 & 8 & 13 & \multirow{4}{*}{13,027} & \multirow{4}{*}{0,043} \\
\hline 6 a 10 años & 1 & 2 & 6 & 10 & 5 & 8 & & \\
\hline 11 a 15 años & 2 & 3 & 0 & 0 & 0 & 0 & & \\
\hline 16 años a más & 0 & 0 & 5 & 8 & 1 & 2 & & \\
\hline \multicolumn{9}{|l|}{ Número de pacientes asignados } \\
\hline 2 pacientes & 8 & 13 & 14 & 23 & 4 & 7 & \multirow{4}{*}{5,574} & \multirow{4}{*}{0,473} \\
\hline 3 pacientes & 4 & 7 & 12 & 20 & 6 & 10 & & \\
\hline 4 pacientes & 0 & 0 & 2 & 3 & 1 & 2 & & \\
\hline 5 pacientes & 0 & 0 & 6 & 10 & 3 & 5 & & \\
\hline \multicolumn{9}{|l|}{ Turnos de Trabajo } \\
\hline Fijo & 2 & 3 & 5 & 8 & 1 & 2 & \multirow{2}{*}{0,635} & \multirow{2}{*}{0,728} \\
\hline Rotativo & 10 & 17 & 29 & 48 & 13 & 22 & & \\
\hline \multicolumn{9}{|l|}{ Trabaja en otra Institución } \\
\hline $\mathrm{Si}$ & 10 & 17 & 20 & 33 & 3 & 5 & \multirow{2}{*}{10,468} & \multirow{2}{*}{0,005} \\
\hline No & 2 & 3 & 14 & 23 & 11 & 18 & & \\
\hline
\end{tabular}

Al relacionar las variables, los datos muestran que no existe relación entre factores personales y actitud hacia la muerte en las enfermeras de UCI (tabla 3), pero sí entre factores laborales y actitud hacia la muerte, específicamente la condición laboral (nombrado/contratado), el tiempo que labora en el servicio (número de años) y trabajar en otra institución (tabla 4).

\section{DISCUSIÓN}

En el análisis de los factores personales y laborales, los resultados muestran que los profesionales de enfermería estaban en la etapa adulta joven, mujeres de preferencia y con estudios de especialidad en cuidados intensivos (UCI), profesando mayoritariamente la religión católica (tabla 1). Como es propio en esta etapa, la persona está cimentando su estabilidad económica y familiar, por lo que necesita mantener una vida productiva que le ayude a consolidar sus aspiraciones y desarrollar su potencial humano; observándose, por tanto, que más del $50 \%$ de enfermeras tiene hijos y que realiza una doble jornada laboral durante la semana (tabla 2). Perfil semejante a los reportados por Menor-Segura et al. ${ }^{22}$ en enfermeras que laboran en unidad de cuidados intensivos. 
Por otro lado, es innegable que enfermería continúa siendo una carrera profesional eminentemente femenina, ciertamente por ser una carrera con alta dosis de sensibilidad humana y las mujeres suelen estar más asociadas a las características de amor, creatividad, dulzura, minuciosidad, bondad, ternura y arte para el cuidado; lo que no niega que los varones también puedan realizar acciones de cuidado. Encuestas internacionales afirman que en el mundo hay más de 16 millones de profesionales en este sector sanitario y que el $80 \%$ son enfermeras. ${ }^{23}$ Solo en Estados Unidos de los 2.25 millones de enfermeras registrados a fines del siglo XX, más del $95 \%$ eran mujeres. ${ }^{24}$

En el 2013 había un total de 69264 profesionales enfermeras en el Perú, distribuidas en los distintos niveles de atención y el 89,8\% era mujer; estimándose que por cada 10 mil habitantes existían solo 4 profesionales de enfermería, ${ }^{24}$ muy por debajo de lo recomendado por la OMS. ${ }^{25}$

La mayoría de los profesionales de enfermería registró que trabaja bajo la condición de Contrato Administrativo de Servicio-CAS (tabla 2), que es una modalidad de contrato privado institucionalizado en las entidades públicas y que se rige bajo sus propias normas, tal y como se describe en el Decreto legislativo 1057, cuya aprobación fue el 2008. ${ }^{23,26}$ Menor-Segura et al. ${ }^{22}$ reportan que la mayoría de enfermeras trabaja bajo esta modalidad, caracterizada por una alta restricción de los beneficios laborales versus una remuneración más elevada, que puede ser atractiva al inicio del ejercicio laboral, pero que al final no proporciona satisfacción, creando angustia e inestabilidad por la probabilidad o no de renovarse el contrato.

Sobre el tiempo que labora en el servicio, en general se encuentran en los primeros años, lo que condice con la edad de las enfermeras, manteniendo un número coherente en la relación enfermera-paciente (tabla 2). Situación que favorece el cuidado individualizado y minucioso al paciente, otorgando el tiempo necesario y oportuno para sus necesidades, no solo físicas sino también espirituales; sobre todo, por la condición de sufrimiento que experimentan al enfrentar una enfermedad terminal. Tanyi, citado por Velosa y Pérez, ${ }^{27}$ refiere que la espiritualidad es un componente inherente al ser humano, subjetivo, intangible y multidimensional, que le conduce a indagar sobre el significado y el propósito en la vida, la cual puede estar o no relacionada con la religión. En tal sentido, debe ser abordada como componente del cuidado holístico al ser humano.
Perspectiva que contrasta con el predominio de una actitud indiferente y de aparente rechazo por parte del profesional de enfermería hacia la condición terminal de la vida (gráfico 1). Llama la atención en el análisis por dimensiones (gráfico 2) la denominada dimensión "salida", en la que un tercio de profesionales acepta la muerte como un hecho natural, pero solo como forma de escape al sufrimiento, ya que en la dimensión "pasaje" la mayoria (rechazo + indiferencia) parece no confiar mucho en la trascedencia o la felicidad despues de la muerte; evidenciandose temor ante la proximidad de la misma (dimensión temor) y miedo de involucrarse emocionalmente con el dolor que enfrenta la familia del paciente (dimensión perspectiva profesional)

En los resultados se identifican que la doble carga laboral que desempeña el profesional de enfermería y la angustia de la inestabilidad laboral, además del tiempo que lleva en el servicio (tabla 4), están relacionados con esta actitud de indiferencia. Es común escuchar frases de enajenación del profesional de enfermería como yo no soy quien da información pregunte al médico, espere hasta el horario de visita para poder entrar, no puede entrar en este lugar, son normas del hospital, entre otros.

La frialdad con el familiar que está angustiado por la situación de su paciente aumenta la angustia y lo encierra en su dolor. Es necesario enfatizar que el cuidado es la razón de ser de enfermería, sin embargo, éste se fortalece cada vez más de inanición, indiferencia, ensimismamiento y deshumanización ${ }^{10}$

Una explicación que surge al respecto es la insuficiente preparación recibida en la formación de pregrado. Hablar sobre la muerte y la terminalidad en la teoría es en sí difícil y denso, por su complejidad y los diversos enfoques psicológicos, espirituales, religiosos y culturales que intervienen en su concepción e interpretación; para muchos puede ser un tema vetado que solo es cabible cuando se vive una experiencia familiar o cercana. Pasado la etapa universitaria, solo la experiencia clínica con el debido acompañamiento y la especialidad en cuidados paliativos harán de la enfermera una profesional idónea para acompañar el trance de la vida-muerte.

Velosa y Pérez, ${ }^{27}$ citando a Minner, refieren que para acompañar al paciente en esos momentos es necesario saber hablar, escuchar, orar o realizar juntos lecturas religiosas. Las autoras acotan que el cuidado espiritual es ineludible en esos momentos y significa hacer testimonio, facilitar y validar la búsqueda de la 
trascendencia, legitimar y ayudar a interpretar la remiscencia de la propia vida; es estar presente allí, en ese momento, con autenticidad.

Torralba $^{26}$ menciona también que el cuidado espiritual es acción que implica movimiento traducido en alivio, atención, ayuda, comodidad y apoyo. Es la creación de relaciones terapéuticas o de momentos de cuidados trascendentes entre la enfermera-paciente, que se viven a través de la entrevista, el lenguaje verbal y no verbal, el contacto visual, físico y el respeto por los momentos de soledad y silencio, la sensibilidad, la capacidad interpretativa de la información, así como la capacidad de observación, escucha e intuición. ${ }^{27}$

Comportamientos que deben estar alimentados por una actitud favorable, proactiva y sensible por parte del profesional de enfermería. Es posible que las enfermeras sean altamente eficientes en el cuidado físico y fisiológico, pero aun requieren de mayor preparación para involucrarse con el cuidado espiritual, para saber estar presente, saber mirar sin hablar, saber escuchar sin interrumpir, saber consentir que comprende lo inefable que es la transcendencia de la vida-muerte y que se puede confiar en ella. No es fácil esta preparación, como lo reitera Pennintong citado por Velosa y Pérez; ${ }^{27}$ acompañar este momento significa también estar impregnado de los principios éticos que están intrínsecamente ligados al cuidado.

De acuerdo con García, $^{28}$ entre las virtudes necesarias para brindar cuidado resaltan: La empatía, la prudencia, la compasión, el afecto, la coherencia y la escucha; las cuáles no siempre son fáciles de trasmitir en la enseñanza y, por lo tanto, son dejadas de lado frente a la formación técnica procedimental, manejo de equipos y terapia medicamentosa, todo con la intención de garantizar la sobrevida de los pacientes.

Otra explicación sobre la actitud de indiferencia del profesional de enfermería ante el paciente terminal sería, el cansancio físico y mental que enfrentan por la doble carga laboral que desempeñan al trabajar paralelamente en clínicas privadas; además de su inestabilidad laboral por mantener contratos que se renuevan cada cierto tiempo (tabla 4).

Menor-Segura et al. ${ }^{22}$ refieren que el solo hecho de laborar en una unidad de cuidado intensivo implica vivir situaciones de estres, dado que es un lugar dónde pacientes y familiares están sumidos en sufrimiento e incertidumbre, quienes a su vez exigen del profesional alta competitividad para el cuidado y manejo de la tecnología avanzada, lo que ocasiona en la enfermera alta presión laboral y psicoemocional, con graves repercusiones en su desempeño y salud general. Sumado a las excesivas horas de trabajo diario por la doble jornada laboral, acaban en desgaste físico y emocional que se traducen en sentimientos de negatividad y actitud de cinismo en su quehacer.

$\operatorname{Cinta}^{20}$ señala que muchas enfermeras no muestran sentimientos conmovedores cuando enfrentan a la familia de un paciente terminal, olvidándose de sus actitudes humanas y actuando de una manera hermética. Es importante recordar que tanto el paciente como su familia, experimentan sentimientos encontrados que va desde la negación, indignación, depresión y finalmente la aceptación; pero todo esto es un proceso contínuo en la que todos (paciente-familia) silenciosamente claman por ayuda.

Maza et al., ${ }^{2}$ en su estudio realizado con enfermeras de dos grandes hospitales en ConcepciónChile (ciudad y al interior), reportan que las actitudes de las enfermeras fueron negativas, identificándose como factores relacionados la edad (extremas), pobre a regular formación en pregrado sobre la muerte, la edad del primer contacto con el deceso (más tardío) y los años de experiencia profesional. La mayoría de enfermeras refirió que su formación fue inadecuada en todas las áreas, destacando el manejo del dolor, cuidado al moribundo, necesidades de los familiares como cuidadores, control de síntomas, proceso de duelo, cuestiones éticas, entre otros.

Por otro lado, las enfermeras también comentaron que no existía en sus hospitales sistemas de apoyo a los profesionales que enfrentan día a día la muerte; sugiriendo: Consejerías, apoyo psicológico, seminarios y talleres. ${ }^{2}$ Estrategias que deben ser implementadas en las instituciones de salud para brindar soporte a los profesionales y éstos a su vez a los pacientes, en una época en que la deshumanización impera en todos los espacios sanitarios; desde que uno contacta con un servicio de salud puede percibir cuánta falta hace la cordialidad y el carisma, el respeto y la empatía. Características que han sido obnubiladas porque la salud se ha comercializado y porque éstos no forman parte de los indicadores de producción.

Es urgente revisar los currículos de formación en pregrado y posgrado, para introducir claramente temas relacionados con la muerte y la terminalidad, muchos más ahora frente al incremento de las enfermedades crónicas no trasmisibles, que conducen a estados límites de salud en los últimos periodos de vida y que claman por un cuidado verdaderamente holístico. Sánchez, citado por Velosa y Pérez., ${ }^{27}$ refiere que el reto es lograr reconocer al paciente como la totalidad del ser humano 
que es, donde su bienestar solo se alcanza cuando su espiritualidad se logra involucrar en el cuidado, proceso complejo que trasciende la enfermedad misma y que integra la mente, cuerpo y espíritu.

Una limitación del estudio es su muestreo no probabilístico, su tamaño muestral y la especificidad de su población, profesionales que asisten a personas en estado crítico y altamente inestables y en muchas ocasiones sedadas. Entre tanto, situaciones de terminalidad y muerte se presentan cotidianamente en todas las áreas del sistema sanitario, debiendo las enfermeras y los médicos estar adecuadamente preparados y recibir constantemente soporte por parte de las instituciones de salud.

Considerando los escasos estudios sobre el tema investigado se sugiere realizar nuevas investigaciones o replicar el estudio en otras poblaciones, a fin de consolidar los hallazgos de este estudio y fomentar cambios en el contexto de cada hospital/centro sanitario.

Así siendo, se concluye que existe relación significativa entre algunos factores laborales y la actitud

\section{Correspondencia:}

Mirabel García Rojas

Correo electrónico: mirabelgr@ hotmail.com

\section{REFERENCIAS BIBLIOGRÁFICAS}

1. Bayes R, Limonero J, Romero E. ¿Qué puede ayudarnos a morir en paz?. Med Clic [serie de internet]. 2000 [citado 10 mar 2016]; 115: 579-582 Disponible en: http://www.elsevier.es/es-revistamedicina-clinica-2-articulo-que-puede-ayudarnosmorir-paz-S0025775300716307

2. Maza CM, Zavala GM, Merino EJM. Actitud del Profesional de enfermería Ante la muerte de pacientes. Cienc enferm [serie de internet]. Abr 2009 [citado 24 ago 2014]; 15(1): 39-48. Disponible en: http://www.scielo.cl/pdf/cienf/v15n1/art06.pdf

3. Ascencio HL, Allende PSR, Verástegui AE. Creencias, actitudes y ansiedad ante la muerte, en un equipo multidisciplinario de cuidados paliativos oncológicos. Psicooncología [serie de internet]. 2014 [citado 10 set 2016]; 11(1): 101-115. Disponible en: http://revistas.ucm.es/index.php/PSIC/article/view/4 $\underline{4920 / 42836}$

4. Tomas-Sabado J, Gomez-Benito J. Variables relacionadas con la ansiedad ante la muerte. Rev Psicol Gral Aplic [serie de internet]. 2003 [citado 15 abr 2016]; 56(3):257-279. Disponible en: https://www.researchgate.net/publication/28169588_ Variables_relacionadas_con_la_ansiedad_ante_la_m $\underline{\text { uerte }}$ hacia la muerte en las enfermeras de UCI, como la condición laboral (nombrado/contratado), el tiempo que labora en el servicio (número de años) y trabajar en otra institución; destacando una actitud de indiferencia ante la muerte de los pacientes terminales por parte de las enfermeras. Resultados que son preocupantes porque muestran claramente la poca preparación que tienen las enfermeras para brindar cuidado en el final de la vida a los pacientes, caracterizado por el poco conocimiento que tienen de la muerte como trascendencia y miedo de enfrentarla por no saber cómo lidiar con ese proceso.

\section{Declaración de financiamiento y de conflictos de interés:}

El estudio fue financiado por la autora, declara no tener conflictos de interés.

\section{Contribución de auditoría:}

García RM: Concepción y diseño del estudio, recolección de datos, análisis e interpretación de los resultados y aprobación de la versión que será publicada.

5. Grau AJ, Llantá AMC, Massip PC, Chacón RM, Reyes MMC, Infante PO, et al. Ansiedad y actitudes ante la muerte: revisión y caracterización en un grupo heterogéneo de profesionales que se capacitan en cuidados paliativos. Pensamiento Psicológico [serie de internet]. Ene-jun 2008 [citado 17 oct 2016]; 4(10): 27-58. Disponible en: http://www.redalyc.org/pdf/801/80111670003.pdf

6. Organización Mundial de la Salud. Programas Nacionales de Lucha Contra el Cáncer. Directrices sobre Política y Gestión [internet]. Ginebra: OMS; 2002 [citado 15 oct 2015]; p.26. Disponible en: http://www.who.int/cancer/media/en/423.pdf

7. Sepúlveda BC. Perspectiva de la OMS para el desarrollo de cuidados paliativos en el contexto de programas de control de cáncer en países en vías de desarrollo [Internet]. OMS: Ginebra; 2002 [citado 22 set 2014]. p.08. Disponible en: http://paliativossinfronteras.org/wpcontent/uploads/LA-OMS-Y-LOS-CP-EN-PAISESEN-DESARROLLO.pdf

8. Oviedo SSJ, Parra FFM, Marquina VM. La muerte y el duelo. Enfermería Global [serie de internet]. Feb 
2009 [citado 28 oct 2014]; 15:[cerca 09 laudas]. Disponible en: http://revistas.um.es/eglobal/article/view/50381/483 $\underline{01}$

9. Burgos AM, Muñoz SD. Enfermeria y el paciente en situación terminal. Rev Enferm Glob [serie de internet]. Jun 2009 [citado 12 ago 2015]; 16: [cerca 09 laudas]. Disponible en: http://scielo.isciii.es/pdf/eg/n16/reflexion3.pdf

10. Sánchez HB. Dimensión espiritual del cuidado de enfermería: en situación de cronicidad y muerte [internet]. Bogotá: Universidad Nacional de Colombia; 2004 [citado 21 ago 2014]. Disponible en:

https://core.ac.uk/download/files/334/11052362.pdf

11. Álvarez J. Actitud del enfermero (a) hacia el proceso de la muerte de sus pacientes en el Servicio de Emergencia de Adultos del Hospital Nacional Edgardo Rebagliati Martins Lima. 2010 [tesis especialidad]. [internet]; Lima-Perú: Universidad Nacional Mayor de San Marcos; 2011 [citado $20 \mathrm{feb}$ 2015]. p.78. Disponible en $\underline{\text { http://bases.bireme.br/cgi- }}$

bin/wxislind.exe/iah/online/?IsisScript=iah/iah.xis\& $\underline{\mathrm{src}=\text { google } \& \text { base }=\text { LILACS\&lang }=p \& \text { nextAction }=1 \mathrm{n}}$ $\underline{k} \&$ exprSearch $=726020$ \&indexSearch $=I D$

12. Flores Y. Actitud del profesional de enfermería hacia el cuidado del paciente moribundo en el servicio de emergencias del Hospital Nacional Hipólito Unanue: Lima. 2013 [tesis especialidad]. [internet]. Lima-Perú: Universidad Nacional Mayor de San Marcos; 2014 [citado 15 febrero 2015]. p.82. Disponible

en: http://ateneo.unmsm.edu.pe/ateneo/bitstream/123456 789/4264/1/Flores_Benites_Yuliana_Carina_2014.p df

13. Pastrana T, De Lima L, Wenk R. Eisenchlas J, Monti C, Rocafort J, et alt. Atlas de Cuidado Paliativos de Latinoamérica ALCP [Internet]. IAPCH Press: Houston; 2012 [citado 30 ago 2014]. p.05. Disponible en: http://cuidadospaliativos.org/uploads/2012/10/atlas/1 9_Peru.pdf

14. Ariza OC, Daza CR. Calidad del cuidado de enfermería al paciente hospitalizado Actualización en enfermería [serie de internet]. 2005 [citado: 20 agosto 2014]; 8(3):8-14. Disponible en: https://encolombia.com/medicina/revistasmedicas/enfermeria/ve-83/enfermeria8305-calidad/

15. Rivera C. La humanización de la vida: una utopía posible "la enfermedad en el tiempo de la enfermedad". Lima- Perú: 1 edición. Editores S.A.C; 2008.

16. Quintero LMC. Espiritualidad y afecto en el cuidado de enfermería. Libros de Enfermería. Colombia [internet]. 2000 [citado 18 agosto 2014]. p.10. Disponible en: http://biblio3.url.edu.gt/Publi/Libros/CyPdeEnferme ria/03.pdf.

17. Hospital Nacional Dos de Mayo. Historia y Tradición. 2012 [internet]. [citado el 11 nov 2014]. Disponible en: http://hdosdemayo.gob.pe

18. Hernández CG, González GVM, Fernández ML, Infante PO. Actitud ante la muerte en los Médicos de Familia. Rev Cubana Med Gen Integr [serie de internet]. Feb 2002 [citado 03 agos 2016]; 18(1):2232. Disponible en: http://scielo.sld.cu/pdf/mgi/v18n1/mgi04102.pdf

19. Chloé DM, Salovey P. Death Attitudes and Selfreported Health- relevant Behaviors. Rev Journal of Health Psychology [serie de internet]. Oct 1996 [citado 10 oct 2016]; 1(4): 441-453. Disponible en: http://hpq.sagepub.com/content/1/4/441.short

20. Cinta SA. Actitud Tanatológica de enfermería con pacientes en etapa terminal [tesis de pregrado]. [internet]. Veracruz-México: Universidad Veracruzana; 2009 [citado 15 ago 2014]. p.75. Disponible

en: http://cdigital.uv.mx/bitstream/123456789/30064/1/a ngelicacintasan.pdf.

21. Vizcarra A. Factores Asociados a la Actitud del Personal de Enfermería ante la Muerte de los Pacientes en el Hospital Hipólito Unanue. Tacna. Año 2012 [tesis de licenciatura]. [internet]. Perú: Universidad Nacional Jorge Basadre Grohmann; 2013 [citado 17 ago 2014]. p.78. Disponible en: http://tesis.unjbg.edu.pe:8080/bitstream/handle/unjb g/228/113_2013_Vizcarra_Oros_AP_FACS_Enferm eria_2013.pdf?sequence $=1$

22. Menor-Segura MR, Díaz-Sotelo RM, FernándezHenríquez LO. Factores estresores y síndrome de Burnout en enfermeras de cuidados intensivos en dos hospitales de EsSalud nivel III-2 Lima Metropolitana. Cuid salud [serie de internet]. Enejun 2015 [citado 05 oct 2016]; 2(1): 137-147. Disponible en: http://eeplt.edu.pe/revista/index.php/Salud/article/vie $\underline{w} / 35 / 31$

23. EFE Salud. El director mundial de la enfermería reivindica el valor de la profesión [Internet]. MadridEspaña: EFE Salud; 2014 [actualizado s.f.; citado e 08 mar 2016], Disponible en: 
http://www.efesalud.com/director-general-mundialenfermeria-reivindica-valor-profesion/

24. Pérez A. Día Internacional de la Enfermera. Infomed Salud Vida. 2016 [internet]. [citado 02 mar 2016]. Disponible en: http://www.sld.cu/saludvida/temas.php?idv=4455

25. INEI. Recursos Humanos de salud. 2016. [Internet]. Lima-Perú: INEI; 2016 [actualizado s.f.; citado 05 mar 2016]. Disponible en: https://www.inei.gob.pe/estadisticas/indicetematico/health-human-resources/

26. OPS/OMS. La OMS/OPS destaca la necesidad de formar más profesional de enfermería en América Latinas y el Caribe [internet]. OMS/OPS: Washington DC; mayo 2015 [actualizado 12 may 2015; citado 10 oct 2015]. Disponible en: http://www.paho.org/hq/index.php?option=com_con tent\&view=article\&id=10947:2015-pahowhohighlights-need-train-more-nursingpersonnel\&Itemid=1926\&lang=es

27. Velosa GMM, Pérez GB. La espiritualidad: componente del cuidado de enfermería. Hallazgos [revista de internet]. Ene-jun 2009 [citado 10 oct 2016]; 6(11):151-162. Disponible en: http://www.redalyc.org/pdf/4138/413835199009.pdf

28. Torralba R F. Ética del cuidar fundamentos contextos y problemas. Mapfre, España; 2002.

29. García M L. La ética del cuidado y su aplicación en la profesión de enfermería. Acta Bioethica [serie de internet]. 2015 [citado 02 oct 2016]; 21(2):311-17. Disponible en: http://www.scielo.cl/pdf/abioeth/v21n2/art17.pdf 